\title{
Aplicación del simulador Aspen HYSYS en la resolución de problemas del ciclo Rankine regenerativo con recalentamiento intermedio
}

\author{
Segundo A. Vásquez ${ }^{1 *}$, Ada P. Barturén ${ }^{1}$ y Felix M. Carbajal ${ }^{2}$ \\ (1) Grupo de Investigación CIMAYDS, Facultad de Ing. Química e Industrias Alimentarias de la Universidad Nacional \\ Pedro Ruiz Gallo, Lambayeque-Perú (correo-e: svasquezll@unprg.edu.pe; abarturen@unprg.edu.pe). \\ (2) Departamento de Engenharia de Energia, Fac. Gama, St. Leste Projecão A - Gama Leste, Universidad de Brasilia, \\ DF 72444-240, Brasilia-Brasil (correo-e: fcarbajal@unb.br).
}

Recibido Dic. 5, 2019; Aceptado Feb. 5, 2020; Versión final Mar. 29, 2020, Publicado Jun. 2020

\begin{abstract}
Resumen
Este trabajo se enfocó en validar el simulador Aspen HYSYS para resolver dos casos representativos del ciclo Rankine regenerativo con recalentamiento intermedio. La interpretación del ciclo Rankine por el método manual es complejo y requiere extenso tiempo. El primer caso comprendió el ciclo con un calentador abierto y uno cerrado con una extracción de vapor en la turbina de baja presión. El segundo caso abarcó el ciclo con tres extracciones de vapor, dos en la turbina de alta presión, una en la turbina de baja presión y tres calentadores abiertos. La validación del simulador Aspen HYSYS alcanzó discrepancias del $1.2 \%$ con relación a los cálculos manuales. El simulador Aspen HYSYS mostró versatilidad para evaluar diversas variables que pueden afectar la eficiencia térmica del ciclo. En conclusión, el simulador Aspen HYSYS puede ser utilizado para la enseñanza y comprensión del ciclo Rankine.
\end{abstract}

Palabras clave: ciclo Rankine; ciclo Rankine regenerativo; ciclo de potencia de vapor; Aspen HYSYS

\section{Application of the Aspen HYSYS simulator to solve problems of the regenerative Rankine cycle with intermediate reheating}

\begin{abstract}
This work focused on validating the Aspen HYSYS simulator for interpreting the regenerative Rankine cycle with intermediate overheating. Manual methods for interpreting the Rankine cycle are complex and are time consuming. Two representative cases were examined to validate the Aspen HYSYS simulator. The first case had a Rankine cycle with: 1) an open heater and 2) a closed heater with steam extraction in the low pressure turbine. The second case had three open heaters and three steam extractions: two in the high pressure turbine and one in the low pressure turbine. Validation of the Aspen HYSYS simulator showed discrepancies of $1.2 \%$ in relation to manual calculations. The Aspen HYSYS simulator was versatile for evaluating variables that can affect the thermal efficiency of the cycle. In conclusion, the Aspen HYSYS simulator can be employed for teaching and for analyzing the Rankine cycle.
\end{abstract}




\section{INTRODUCCION}

El ciclo Rankine es considerado un tópico esencial en el área de la termodinámica. Es descrito como la conversión de calor en trabajo mecánico y está ampliamente presente en las centrales de conversión de energía eléctrica (Wiser, 2000). Debido a su importancia y consolidación del ciclo Rankine, los alumnos de ingeniería necesitan entender e interpretar con precisión los fenómenos que ocurren durante este ciclo. El ciclo Rankine utiliza comúnmente el agua como fluido de referencia, para la generación de electricidad donde ocurren diversos cambios de su estado físico (Rashidi et al., 2014; Arce y Viera, 2017). El ciclo Rankine ideal se desarrolla en cuatro etapas: 1) compresión isentrópica en la bomba, 2) adición de calor isobárica en la caldera, 3) expansión isentrópica en la turbina y 4) rechazo isobárico de calor en el condensador (Prosun, 2015; Arce y Viera, 2017).

Entretanto, a pesar de su consolidación, en los últimos años algunas modificaciones fueron realizadas al ciclo Rankine con el objetivo de mejorar su eficiencia térmica. Entre estas, tenemos el ciclo Rankine regenerativo. En este ciclo, el calentamiento del agua de entrada es utilizando el vapor extraído de una o varias zonas de la turbina, con la finalidad de aprovechar parte de la energía que de otra forma se perderían en el condensador (Sahu et al., 2017). Lo interesante de esta modificación al ciclo Rankine convencional es que al adicionar los calentadores abiertos de agua de alimentación, el calor se transfiere directamente del vapor al agua de alimentación mezclándose las dos corrientes de fluido. En este mismo ciclo, los calentadores cerrados de agua de alimentación, los fluidos circulan a través del calentador sin mezclarse. Por lo tanto, considerando las modificaciones mencionadas, el ciclo Rankine regenerativo mejoró la eficiencia térmica del ciclo.

En esta dirección, otra destacable modificación al ciclo Rankine y que mejora significativamente la eficiencia térmica del ciclo entre 4 a 5\%, es el ciclo Rankine regenerativo con la inclusión del recalentamiento intermedio (Valencia et al., 2017). Considerando esta modificación al ciclo Rankine, Rashidi et al. (2014) alcanzaron una eficiencia térmica del $49.15 \%$ en una planta de potencia de vapor. En esta investigación abordaron el ciclo Rankine regenerativo con doble recalentamiento que consistió en 3 turbinas, siendo una de alta, una de media y una de baja presión. Adicionalmente ese ciclo contempló varias extracciones de vapor con seis calentadores cerrados de agua de alimentación. En cuanto, Valencia et al. (2017) obtuvieron una eficiencia térmica de $46.2 \%$ para una planta de vapor que estaba integrada de dos calentadores cerrados, un calentador abierto, tres turbinas con tres extracciones de vapor y un recalentamiento intermedio.

No obstante, hacer el análisis termodinámico y determinar la eficiencia térmica del ciclo Rankine regenerativo con recalentamiento intermedio por el método manual. Requiere innúmeras interpolaciones para determinar las propiedades termodinámicas del agua en diferentes estados físicos tales, como; las entalpías, entropías y volúmenes específicos usando las tablas de vapor o el diagrama de Mollier. Adicionalmente, se necesita extenso tiempo para hacer uso de estas propiedades termodinámicas en las ecuaciones de balances de transferencia, así como, para determinar: la carga de calor de los equipos involucrados, el trabajo neto del ciclo, las fracciones de vapor extraído de la turbina o la realización de un análisis de sensibilidad como la variación del incremento de la temperatura y presión a la entrada de la turbina de alta presión sobre la eficiencia térmica del ciclo manteniendo la presión constante en el condensador. Esta resolución de problemas obliga hacer cálculos repetitivos, perdiendo un tiempo importante para la interpretación de los resultados.

Por lo tanto, un análisis termodinámico exhaustivo de los ciclos de vapor y el uso de herramientas computacionales pueden contribuir, para mejorar la interpretación de los ciclos (Méndez-Cruz et al., 2019). En esta dirección, (Valderrama y Véliz, 2003; Guerra et al., 2005) realizaron la simulación de ciclos de vapor con cogeneración utilizando herramientas computacionales. De esta forma, el uso de herramientas computacionales en el análisis del ciclo Rankine se convirtió en una tendencia, entre estas tenemos: el programa Ciclos 2005 desarrollado en Visual Basic (Mogollón et al., 2007), interfaces gráficas implementados en MATLAB (Marwan et al., 2015; Arce y Viera, 2017). También, el programa The Power Cycle V2.0 (Duarte et al., 2018; Valencia et al., 2018) y recientemente la incorporación de Aspen HYSYS.

El uso del simulador Aspen HYSYS es interesante, porque es direccionado para fines comerciales. No obstante, Aspen HYSYS es una herramienta informática muy poderosa en las áreas de ingeniería, investigación científica, innovación, educación. Se caracteriza por su amigable interface, la interactividad al momento de modificar las condiciones del proceso en cualquier instante y realiza los cálculos mostrando los resultados automáticamente. Investigaciones demuestran la capacidad del simulador Aspen HYSYS, como su uso en el análisis del ciclo de potencia de vapor (Brammer y Hessami, 2008; Ibrahim et. al., 2011). También, su uso en la integración de diversos sistemas de energía (Liu y Karimi, 2019) y en la evaluación exergética de un ciclo Rankine regenerativo con calentador abierto (Arrieta et al., 2018). 
En este contexto, este trabajo tuvo como objetivo la aplicación del simulador Aspen HYSYS en el desarrollo y la interpretación de problemas del ciclo Rankine regenerativo con recalentamiento intermedio para fines académicos. Dos casos de estudios fueron abordados. El primero enfocó el ciclo Rankine regenerativo con un recalentamiento intermedio con calentador cerrado de agua de alimentación, una turbina de alta presión y una turbina de baja presión con extracción de vapor. Las turbinas y las bombas trabajan con una eficiencia isentrópica de $88 \%$. Entretanto, el segundo caso comprendió el ciclo Rankine regenerativo ideal con recalentamiento intermedio compuesto de: dos turbinas, la de alta presión realiza dos extracciones de vapor y la turbina de baja presión una extracción de vapor. Adicionalmente este ciclo modificado contempla tres calentadores abiertos de agua de alimentación.

\section{METODOLOGÍA}

Se utilizó el simulador Aspen HYSYS V7.3 (AspenTech, 2011) y el paquete de propiedades de fluidos ASME Steam para el cálculo de las propiedades termodinámicas del agua. La herramienta Spreadsheet del simulador fue usada para realizar los cálculos tales como: el calor de entrada, calor de salida, las fracciones de vapor extraído de las turbinas, los flujos másicos de vapor extraído de las turbinas, el trabajo neto y la eficiencia térmica del ciclo. Para la elaboración del diagrama de flujo y con base con el ciclo Rankine fueron seleccionados los siguientes equipos: caldera (heater), condensador (cooler), turbina (expander), bomba (pump), divisor (tee), intercambiador de calor (heat exchanger) y mezclador (mixer). Las condiciones de operación como presión, temperatura, flujo másico, fracción de vapor de las corrientes para los dos casos seleccionados de la literatura (Cengel y Boles, 2012; Rolle, 2006) se ingresaron a través de la interface Workbook del simulador. En cuanto, para la caldera, el recalentador, el enfriador, el condensador, los calentadores abiertos y cerrados de agua de alimentación se consideró que operan a presión constante y las eficiencias isentrópicas para la turbina y la bomba son el $88 \%$ para el Caso 1 y $100 \%$ para el Caso 2 , respectivamente. Todas estas especificaciones se ingresan a través de sus respectivas ventanas de parámetros.

\section{Caso 1}

La Figura 1 representa una planta de energía a vapor que opera en el ciclo Rankine regenerativo con recalentamiento intermedio. El vapor que proviene de la caldera (corriente 4) ingresa a la turbina de alta presión a $8 \mathrm{MPa}$ y $500{ }^{\circ} \mathrm{C}$ con un flujo de $15 \mathrm{~kg} / \mathrm{s}$ (corriente 5) y se condensa a la presión de $20 \mathrm{kPa}$ en el condensador (corriente 1). A la turbina de baja presión ingresa el vapor recalentado a $3 \mathrm{MPa}$ y $500{ }^{\circ} \mathrm{C}$ (corriente 7) y se extrae vapor de la turbina a $1.0 \mathrm{MPa}$ (corriente 8 ) condensándose por completo en el calentador cerrado de agua de alimentación (corriente 3). Posteriormente se bombea a $8 \mathrm{MPa}$ (corriente 11) antes de que se mezcle en la cámara de mezclado con el agua de alimentación a la misma presión (corriente 10). Todas las turbinas y las bombas operan con una eficiencia isentrópica de $88 \%$. Entretanto, la caldera, el calentador cerrado de agua de alimentación y el condensador tienen caídas de presión despreciable y la cámara de mezclado opera a presión constante. Con base en las consideraciones expuestas, la pregunta del problema es: determinar la temperatura del vapor a la entrada del calentador cerrado de agua de alimentación, el flujo másico del vapor extraído de la turbina para el calentador cerrado de agua de alimentación, el trabajo neto y la eficiencia térmica del ciclo (Cengel y Boles, 2012).

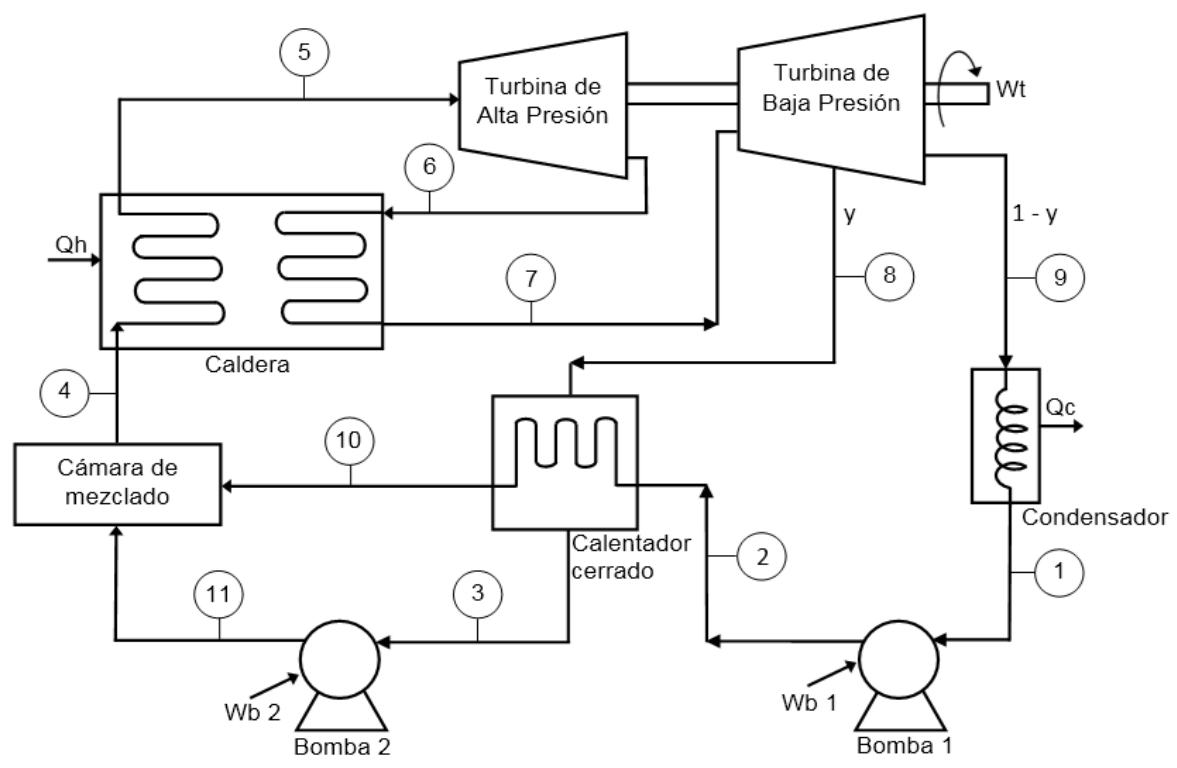

Fig. 1: Ciclo Rankine regenerativo con recalentamiento, adaptada de Cengel y Boles (2012) 
En forma detallada en la Tabla 1 se muestran las especificaciones de las corrientes del caso estudiado que se ingresan a través de la interface Workbook del simulador. Es importante mencionar que no todas las especificaciones y corrientes están presentes, debido que el simulador Aspen HYSYS calculará las especificaciones faltantes de las demás corrientes.

Tabla 1: Especificaciones de las corrientes que se ingresan a través de la interface Workbook Fuente: Caso de estudio 1 (Cengel y Boles, 2012)

\begin{tabular}{|l|l|l|l|l|l|l|l|}
\hline \multirow{2}{*}{ Variables } & \multicolumn{6}{l}{ Corrientes } & \multicolumn{5}{l|}{} \\
\cline { 2 - 9 } & 5 & 7 & 8 & 1 & 10 & 3 & 11 \\
\hline Fracción de vapor & & & & 0 & & 0 & \\
\hline Temperatura, ${ }^{\circ} \mathrm{C}$ & 500 & 500 & & & & & \\
\hline Presión, $\mathrm{kPa}$ & 8000 & 3000 & 1000 & 20 & 8000 & & 8000 \\
\hline Flujo másico, $\mathrm{kg} / \mathrm{s}$ & 15 & & & & & & \\
\hline Composición molar, agua & 1 & & & & & & \\
\hline
\end{tabular}

\section{Caso 2}

El ciclo regenerativo con recalentamiento intermedio contempla dos extracciones de vapor en la turbina de alta presión y una extracción en la turbina de baja presión se muestra en la Figura 2. El vapor que proviene de la caldera (corriente 2) ingresa a la turbina de alta presión a $8274 \mathrm{kPa}$ y $454^{\circ} \mathrm{C}$ con un flujo de $4.54 \mathrm{~kg} / \mathrm{s}$ (corriente 3) donde se realizan dos extracciones de vapor. La primera extracción de vapor es a $4137 \mathrm{kPa}$ (corriente 13). En cuanto que, la segunda extracción de vapor es a $292{ }^{\circ} \mathrm{C}$ y $2758 \mathrm{kPa}$ (corriente 14), condensándose por completo en los calentadores abiertos de agua de alimentación A y B (corrientes 1 y 11). A la turbina de baja presión ingresa el vapor recalentado a $1379 \mathrm{kPa}$ y $427^{\circ} \mathrm{C}$ (corriente 5), de donde se extrae vapor de la turbina a $207 \mathrm{kPa}$ (corriente 15) y se condensa por completo en el calentador abierto de agua de alimentación C (corriente 9). El vapor de escape sale a $7 \mathrm{kPa}$ (corriente 6) condensándose en el condensador (corriente 7), que posteriormente es bombeado a $207 \mathrm{kPa}$ a través de la bomba 4 (corriente 8) antes de ingresar al calentador abierto $\mathrm{C}$. Todas las turbinas y las bombas operan con una eficiencia isentrópica de $100 \%$. La caldera, el recalentador y el condensador tienen caídas de presión despreciable y los calentadores abiertos operan a presión constante. Con base en las consideraciones expuestas, la pregunta del problema es: determinar las fracciones de extracciones de vapor y los flujos másicos de las extracciones de vapor en las turbinas de alta y baja presión, el calor de entrada, el calor de salida, el trabajo neto y la eficiencia térmica del ciclo (Rolle, 2006).

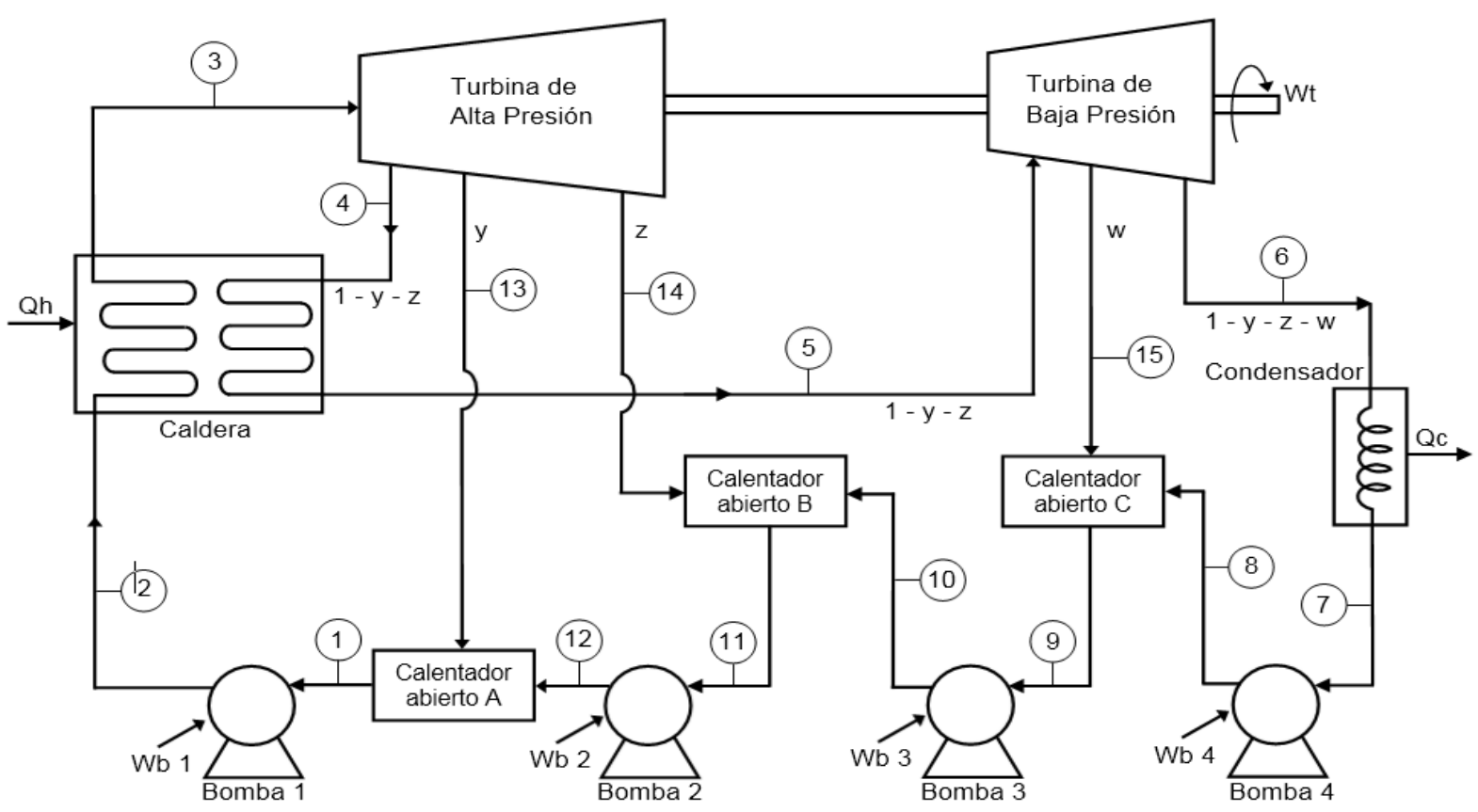

Fig. 2: Ciclo Rankine regenerativo con recalentamiento intermedio y calentadores abiertos, adaptada de Rolle (2006)

En la Tabla 2 se detallada las especificaciones de las corrientes del segundo caso estudiado. Estas especificaciones son ingresadas a través de la interface Workbook del simulador. Es importante mencionar que no todas las especificaciones y corrientes están presentes debido que el simulador Aspen HYSYS calculará las especificaciones faltantes de las demás corrientes. 
Tabla 2: Especificaciones de las corrientes que se ingresan a través de la interface Workbook. Fuente: Caso de estudio 2 (Rolle, 2006)

\begin{tabular}{|c|c|c|c|c|c|c|c|c|c|}
\hline \multirow{2}{*}{ Variables } & \multicolumn{9}{|c|}{ Corrientes } \\
\hline & 1 & 3 & 5 & 7 & 9 & $11 a$ & 13 & 14 & 15 \\
\hline Fracción de vapor & 0 & & & 0 & 0 & & & & \\
\hline Temperatura, ${ }^{\circ} \mathrm{C}$ & & 454 & 427 & & & & & 292 & \\
\hline Presión, kPa & & 8274 & 1379 & 7 & & & 4137 & 2758 & 207 \\
\hline Flujo másico, $\mathrm{kg} / \mathrm{s}$ & & 4.54 & & & & & & & \\
\hline Composición molar, agua & & 1 & & & 1 & 1 & & & \\
\hline
\end{tabular}

\section{Validación de los resultados}

Una vez simulados los diagramas de flujo para los casos 1 y 2 se procede a validar los resultados obtenidos con el simulador Aspen HYSYS. Posteriormente estos resultados son compararlos con los resultados desarrollados manualmente de la literatura (Cengel y Boles, 2012; Rolle, 2006). Para esta comparación se consideró el porcentaje de discrepancia que existe entre el valor teórico de las principales variables, tales como: el calor de entrada, calor de salida, trabajo neto, extracción de vapor, flujo másico de vapor extraído y la eficiencia térmica de los casos de estudio y el valor obtenido con el simulador a través de la ecuación 1. Finalmente se realizaron dos análisis de sensibilidad para el caso 2. En el primer análisis se consideró la variación del incremento de la temperatura $\left(400^{\circ} \mathrm{C}\right.$ a $\left.600^{\circ} \mathrm{C}\right)$ y presión $(7 \mathrm{MPa}$ a $9 \mathrm{MPa})$ a la entrada de la turbina de alta presión sobre la eficiencia térmica manteniendo la presión a $7 \mathrm{kPa}$ en el condensador. En cuanto, el segundo análisis consistió en el efecto de la fracción de vapor de escape en la turbina de baja presión y la eficiencia térmica por efecto del incremento de la presión en el condensador ( $5 \mathrm{kPa}$ a $10 \mathrm{kPa})$.

$\%$ discrepanċa $=\frac{\mid \text { valorteórico-valorAspen HYSYS } \mid}{\text { valorteórico }} * 100$

\section{RESULTADOS Y DISCUSION}

Para el caso 1, la Figura 3 muestra la simulación realizada del ciclo Rankine regenerativo con recalentamiento intermedio generado en el simulador Aspen HYSYS. También, para el Caso 1, la Tabla 3 muestra la comparación de los resultados desarrollados manualmente de la literatura y los resultados obtenidos con el simulador. Para obtener la fracción de separación de la corriente 8 que requiere el divisor se realizó un balance energético en el calentador cerrado de agua de alimentación obteniéndose el valor de 0.1753 el cual se ingresó en la ventana de parámetros del divisor.

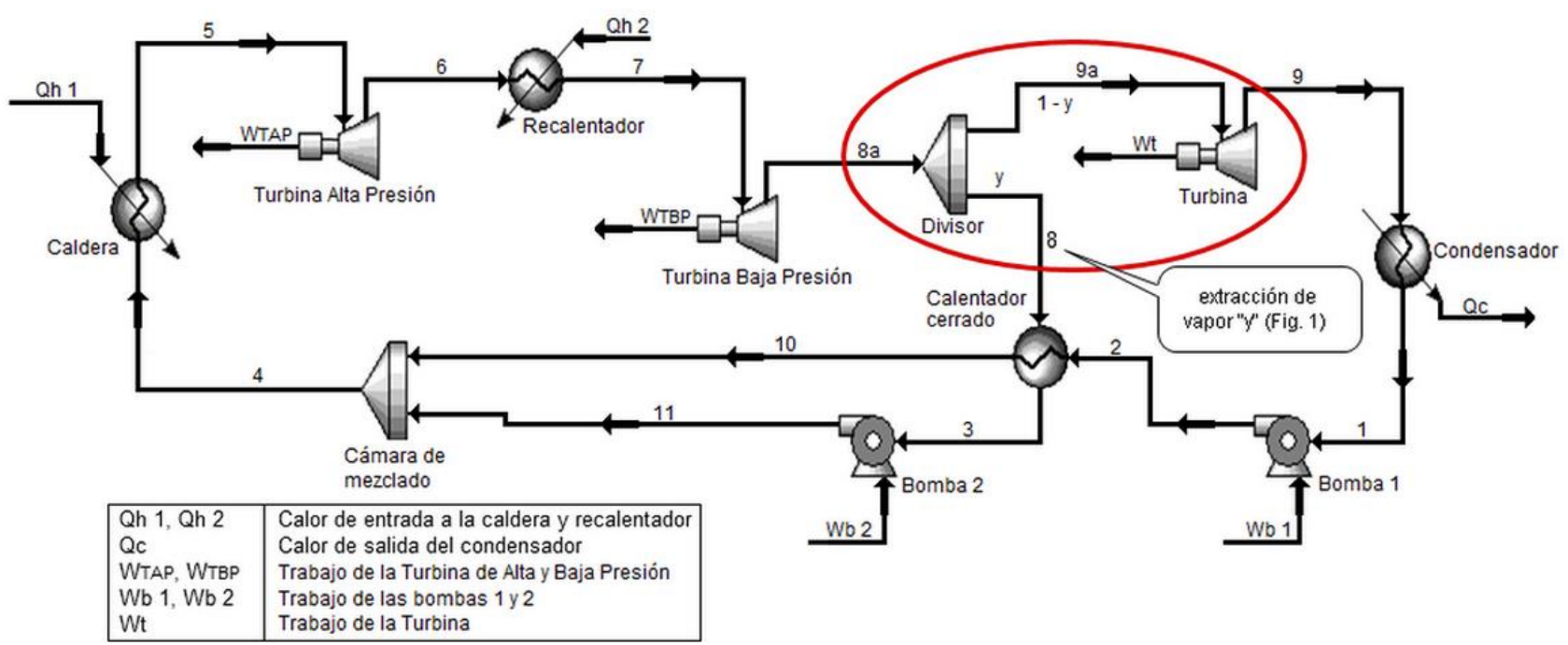

Fig. 3: Diagrama de flujo simulado para el caso 1, adaptada de Aspen HYSYS V7.3

De la Figura 3, se observa el ciclo Rankine regenerativo con recalentamiento intermedio, descrito de la siguiente forma: de acuerdo con Cengel y Boles (2012), la caldera con recalentamiento es representado por un calentador (corrientes 4 y 5). Luego la turbina de alta presión (corrientes 5 y 6 ) y después el recalentador (corrientes 6 y 7). Para representar la extracción de vapor en la turbina de baja presión (corriente 8) se adicionó un divisor (corrientes 9a y 8) y una turbina (corrientes 9a y 9). Posteriormente pasa al condensador (corrientes 9 y 1). Las corrientes $8 a$ y $9 a$ son corrientes auxiliares que se adicionaron para poder simular el ciclo. 
En los resultados presentados en la Tabla 3, se observa las discrepancias que hay entre los resultados obtenidos con el simulador y con los resultados desarrollados manualmente de la literatura (Cengel y Boles, 2012). Esta discrepancia es alrededor del 1.2\% para la determinación del trabajo neto y la eficiencia térmica del ciclo. También, se determinó la fracción de vapor extraído, el flujo másico de vapor extraído y la temperatura para la corriente 8 , el calor total de entrada y el calor de salida mostrando resultados con \% de discrepancia alrededor del $0.7 \%$.

Tabla 3: Comparación de los resultados de la literatura con el simulador para el Caso 1 Fuente: Cengel y Boles (2012); Simulador Aspen HYSYS V7.3

\begin{tabular}{|l|l|l|l|}
\hline Variables & Literatura & Aspen HYSYS & \% discrepancia \\
\hline Fracción de vapor extraído, corriente 8 & 0.1758 & 0.1753 & 0.2844 \\
\hline Temperatura, corriente $8,{ }^{\circ} \mathrm{C}$ & 350 & 349.4 & 0.1714 \\
\hline Flujo másico de vapor extraído, kg/s & 2.64 & 2.630 & 0.3788 \\
\hline Calor total de entrada, Qh, kJ/kg & 2945.2 & 2946 & 0.02716 \\
\hline Calor de salida, Qc, kJ/kg & 1864.8 & 1852 & 0.6864 \\
\hline Trabajo neto, $\mathrm{kW}$ & 16206 & 16400 & 1.1971 \\
\hline Eficiencia térmica, \% & 36.7 & 37.12 & 1.1444 \\
\hline
\end{tabular}

En la Figura 4 se muestra la ventana de la herramienta Spreadsheet del simulador. Se observan todos los valores de las entalpías en las celdas A1 a A10 necesarios, para la realización del balance energético. Esta información relevante ha sido importada a la etiqueta Spreadsheet. Además, se observan las variables calculadas por el simulador (celdas E2 y E4) y las variables calculadas por el usuario que son las celdas E1, E3, E5 a E8. En estas celdas, las ecuaciones son ingresadas en la etiqueta Fórmulas de acuerdo al balance energético que se realizan en el ciclo Rankine.

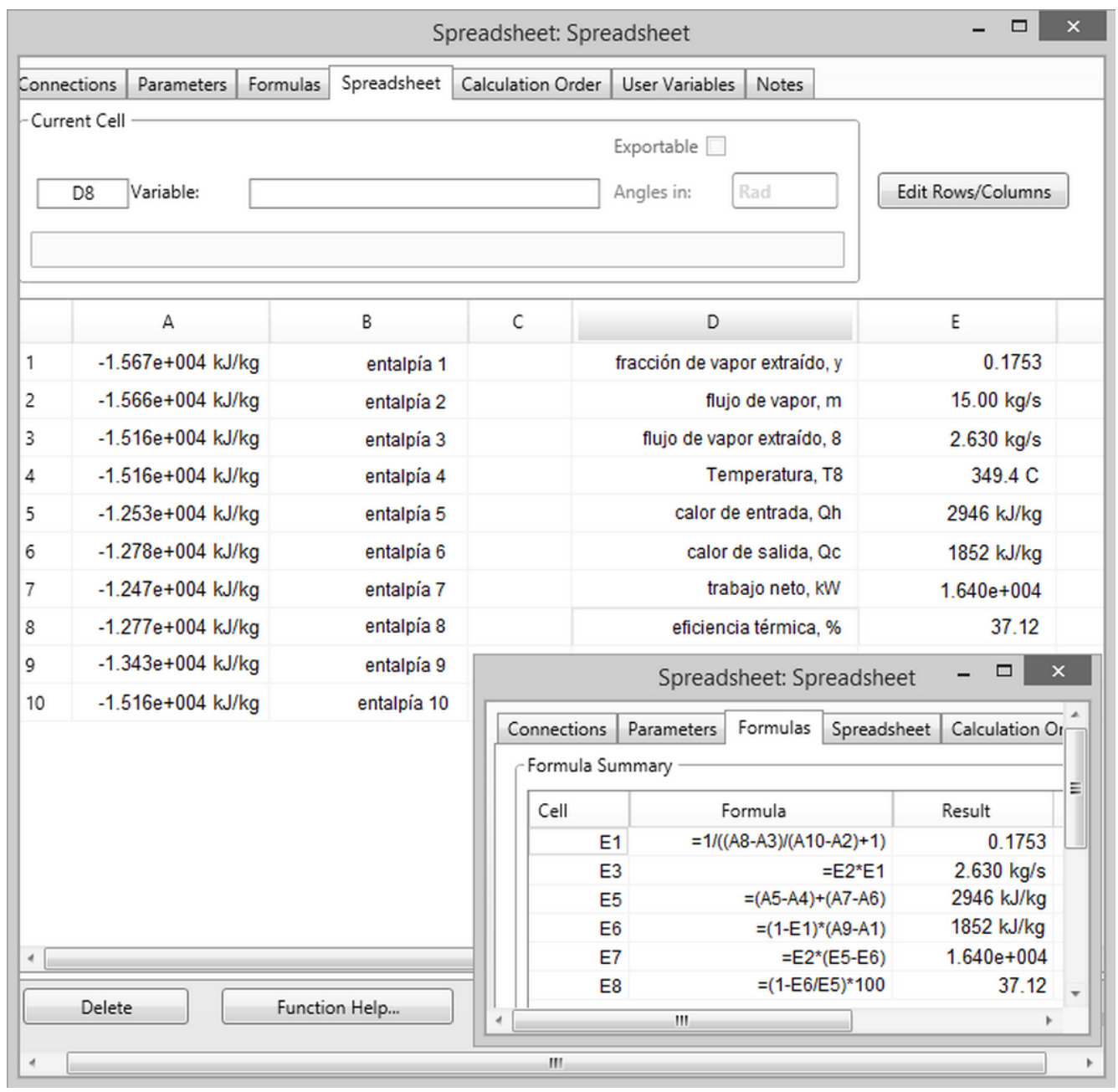

Fig. 4: Resultados obtenidos para el caso 1, adaptada de Aspen HYSYS V7.3 
Para el caso 2, la Figura 5 muestra la simulación realizada del ciclo Rankine regenerativo con recalentamiento intermedio en la caldera (corrientes 4 y 5 ) con dos extracciones de vapor en la turbina de alta presión (corrientes 13 y 14) y una extracción en la turbina de baja presión (corriente 15) con tres calentadores abiertos.

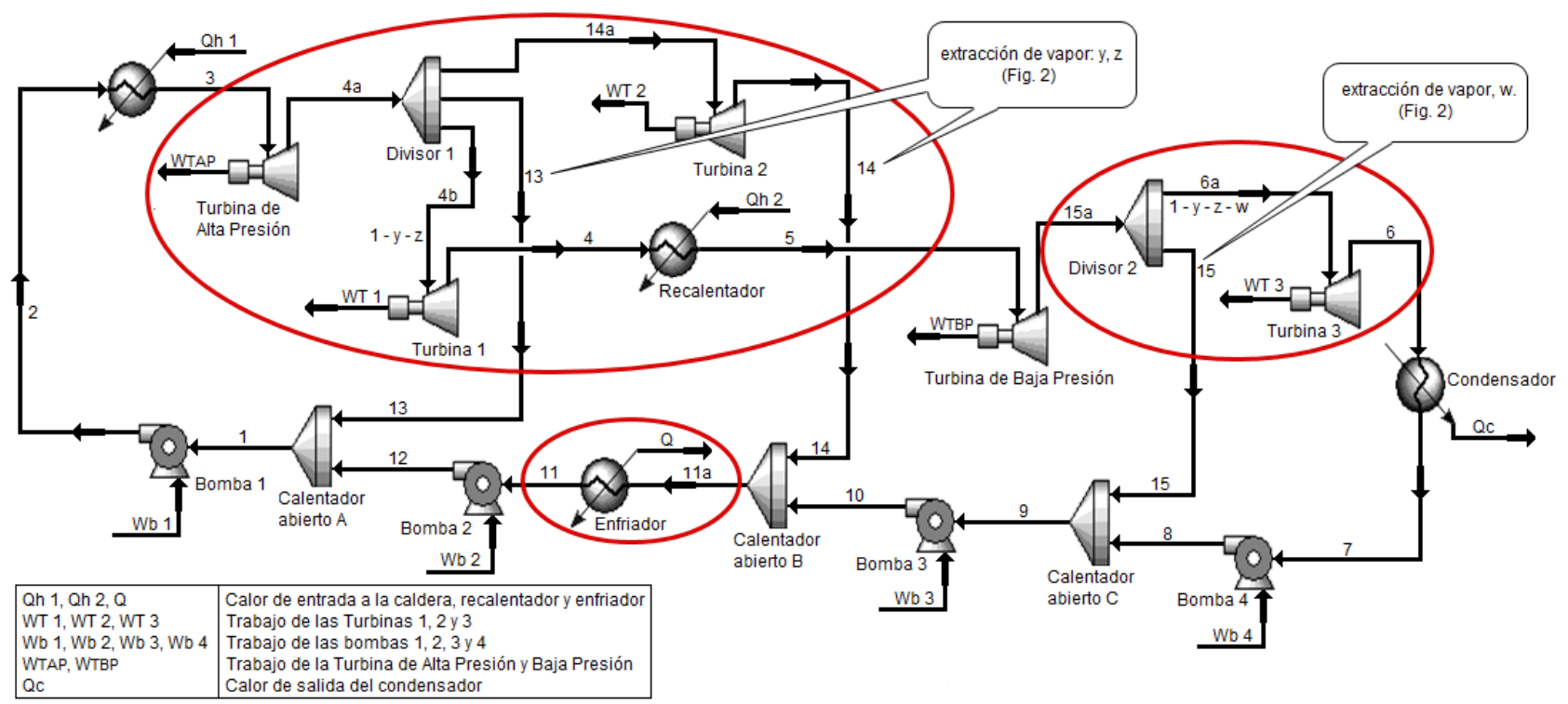

Fig. 5: Diagrama de flujo simulado para el caso 2, adaptada de Aspen HYSYS V7.3

De la Figura 5, se observa el ciclo Rankine regenerativo con recalentamiento intermedio, descrito de la siguiente forma: La caldera con recalentamiento es representado por un calentador (corrientes 2 y 3), la turbina de alta presión (corrientes 3 y $4 a$ ) y de un divisor (corrientes 14a, 13 y $4 b$ ) que representa las extracciones de vapor que salen de la turbina de alta presión son las corrientes 13 y 14, de acuerdo con Rolle (2006). La corriente 4b ingresa a una turbina para obtener los resultados de la corriente 4. Luego esta ingresa al recalentador (corrientes 4 y 5), seguido de otra turbina (corrientes 5 y 15a). La corriente 13 ingresa al calentador abierto A, pero la corriente 14a pasa por otra turbina donde sale la corriente 14 que ingresa al calentador abierto B. Para la extracción de vapor en la turbina de baja presión (corriente 15) se adiciona un divisor (corrientes 6a y 15) y una turbina (corrientes 6a y 6) que luego pasa al condensador (corriente 7). Las corrientes $4 a, 4 b, 6 a, 11 a, 14 a$ y 15a son corrientes auxiliares que se adicionan al diagrama de flujo así como: la adición de las turbinas 1,2 y 3 y los divisores 1 y 2 son para simular el ciclo Rankine. Entre tanto, para las corrientes $4 a$, 4b, 13 y 14a se especificó solamente la presión de la corriente $13(4137 \mathrm{kPa})$ y para las corrientes 6a y 15a se especificó la presión de la corriente $15(207 \mathrm{kPa})$. También se adicionó un enfriador (corrientes 11a y 11). Esto se hace debido a que en el calentador abierto la corriente 11 es líquido saturado. Entretanto, en la simulación la corriente 11 tenía presencia de vapor (0.0156), por lo que se instaló el enfriador con las corrientes 11a y 11, donde la corriente 11 ahora es líquido saturado ingresa a la bomba 2.

En la Figura 6 se observan todos los valores de las entalpías en las celdas A1 a A15 necesarios para la realización del balance energético. Destacamos que estas han sido importadas a la etiqueta Spreadsheet. Además, se observan las variables calculadas por el simulador (celdas E2, E3, E7, E8 y E9). Las variables calculadas por el usuario son las celdas: E4 a E6 y de E10 a E14. En estas las ecuaciones son ingresadas en la etiqueta Fórmulas de acuerdo al balance energético que se realizan en el ciclo Rankine. La celda E1 contiene el valor del flujo másico alimentado desde la interface Workbook que corresponde a la corriente 3.

La comparación de los resultados del caso 2 se presenta en la Tabla 4. Se observan las discrepancias entre los resultados obtenidos con el simulador y los resultados desarrollados manualmente de la literatura (Rolle, 2006). Esta discrepancia fue de alrededor del $0.7911 \%$, para el calor de entrada en el recalentador y $0.1113 \%$ para la eficiencia térmica del ciclo. Por lo tanto, para ambos casos estudiados y simulados con el simulador Aspen HYSYS, los resultados son muy similares a los de la literatura (Cengel y Boles, 2012; Rolle, 2006). Las discrepancias llegaron alrededor del 1.2\% en comparación con el trabajo de Arce y Viera (2015), que mostraron discrepancias alrededor del $2 \%$ al implementar interfaces gráficas con MATLAB. No obstante, la evaluación que realizaron Brammer y Hessami (2008) para ciclos Rankine simples con Aspen HYSYS 2004.1, las discrepancias fueron menores del 1\% para ciclos Rankine ideales y para ciclos Rankine reales son alrededor del 8\%. De igual manera, Valencia et al., (2017) y Arrieta et al., (2018) encontraron discrepancias similares con respecto al trabajo teniendo en cuenta que ellos analizaron hasta ciclos regenerativos con recalentamiento y una extracción. Por lo tanto, resaltamos que los ciclos investigados en 
este trabajo muestran mayor complejidad que los ciclos simulados y encontrados en la literatura. En consecuencia, el simulador Aspen HYSYS se muestra confiable para ser utilizado en el análisis termodinámico del ciclo Rankine regenerativo con recalentamiento intermedio y evaluar el rendimiento de una planta de energía.

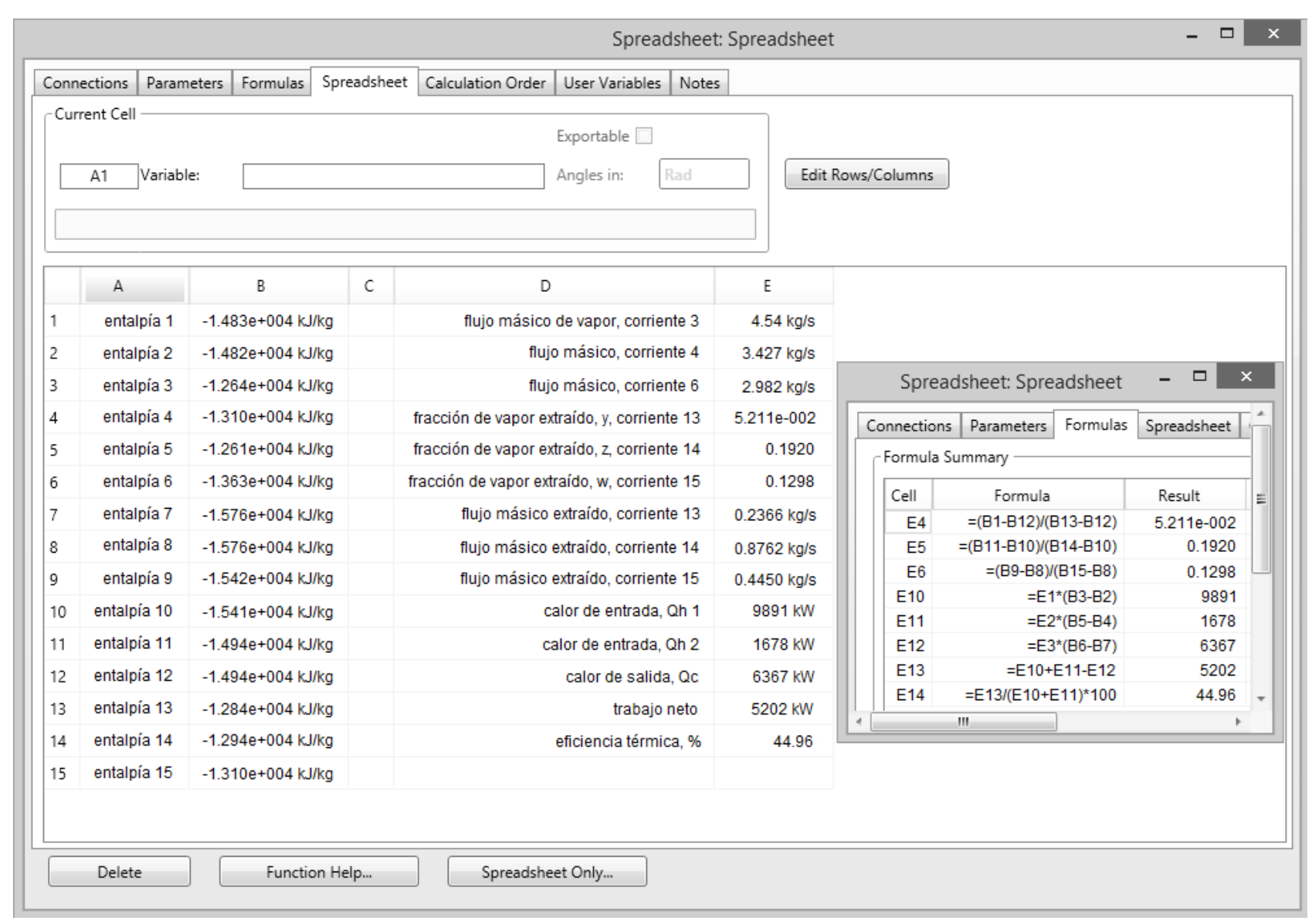

Fig. 6: Resultados obtenidos para el caso 2, adaptada de Aspen HYSYS V7.3

Tabla 4: Comparación de los resultados de la literatura con el simulador para el Caso 2 Fuente: Rolle (2006); simulador Aspen HYSYS V7.3

\begin{tabular}{|l|l|l|l|}
\hline Variables & Literatura & Aspen HYSYS & \% discrepancia \\
\hline Fracción de vapor extraído, y, corriente 13 & 0.05189 & 0.05211 & 0.4240 \\
\hline Fracción de vapor extraído, z, corriente 14 & 0.193 & 0.1920 & 0.5181 \\
\hline Fracción de vapor extraído, w, corriente 15 & 0.13 & 0.1298 & 0.1538 \\
\hline Flujo másico extraído, kg/s, corriente 13 & 0.2359 & 0.2366 & 0.2967 \\
\hline Flujo másico extraído, kg/s, corriente 14 & 0.8754 & 0.8762 & 0.09139 \\
\hline Flujo másico extraído, kg/s, corriente 15 & 0.4459 & 0.4450 & 0.2018 \\
\hline Calor de entrada, Qh 1, kW & 9876.17 & 9891 & 0.1502 \\
\hline Calor de entrada, Qh 2, kW & 1664.83 & 1678 & 0.7911 \\
\hline Calor de salida, Qc, kW & 6358.17 & 6367 & 0.1389 \\
\hline Trabajo neto, kW & 5182.83 & 5202 & 0.3699 \\
\hline Eficiencia térmica, \% & 44.91 & 44.96 & 0.1113 \\
\hline
\end{tabular}

La Figura 7 muestra el efecto de la variación de las temperaturas $\left(400^{\circ} \mathrm{C}\right.$ a $\left.600{ }^{\circ} \mathrm{C}\right)$ y las presiones $(7000$ $\mathrm{kPa}$ a $9000 \mathrm{kPa}$ ) de entrada a la turbina de alta presión manteniendo constante la presión del condensador en $7 \mathrm{kPa}$. La eficiencia térmica del ciclo aumenta de $43.39 \%$ a $48.56 \%$ al variar la temperatura de entrada a la turbina de alta presión desde $400^{\circ} \mathrm{C}$ hasta $600^{\circ} \mathrm{C}$, con una presión de operación de $8274 \mathrm{kPa}$. A mayor temperatura y presión a la entrada de la turbina de alta presión $\left(600^{\circ} \mathrm{C}\right.$, y $\left.9000 \mathrm{kPa}\right)$, la eficiencia térmica aumenta de $43.47 \%$ a $48.79 \%$. En este contexto, las plantas de vapor suelen operar bajo estas condiciones de aumento de la temperatura a una presión adecuada, para incrementar la eficiencia térmica. De esta forma evita el aumento de humedad en el vapor de escape, sin llegar a sobrepasar los $630^{\circ} \mathrm{C}$ (Cengel y Boles, 2012). De llegar a esta última condición conllevaría construir una turbina con aleaciones resistentes. 


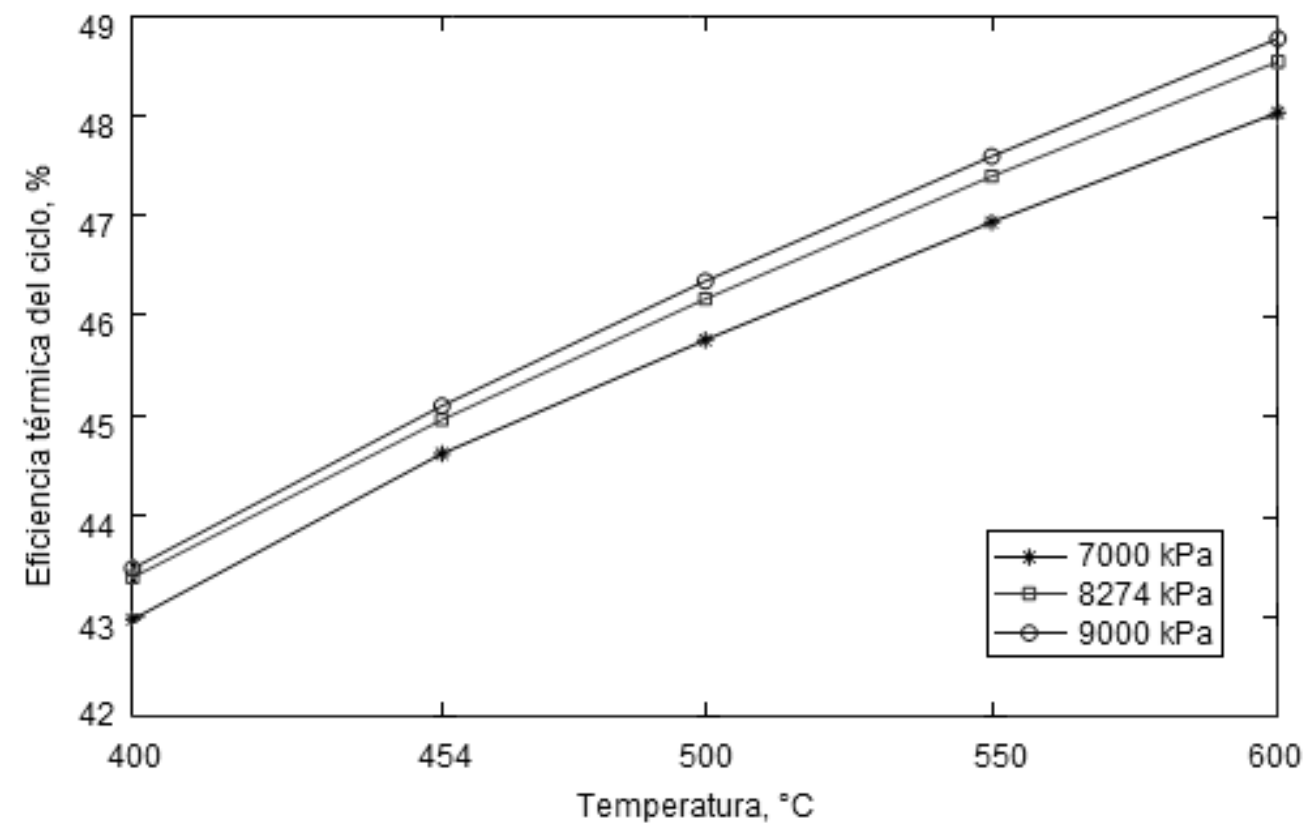

Fig. 7: Variación de la eficiencia térmica del ciclo sobre la temperatura y presión a la entrada de la turbina

En esa línea, la Figura 8 muestra el efecto de incrementar la presión del condensador de $5 \mathrm{kPa}$ hasta 10 $\mathrm{kPa}$ sobre la fracción de vapor a la salida de la turbina de baja presión y la eficiencia térmica del ciclo. Se observa que conforme aumenta la presión del condensador, la fracción de vapor aumenta de 0.8738 a 0.8994 y la eficiencia térmica del ciclo disminuye de $45.91 \%$ a $43.91 \%$. Para las condiciones establecidas para el caso 2, la fracción de vapor es 0.886 con una eficiencia térmica de $44.96 \%$.

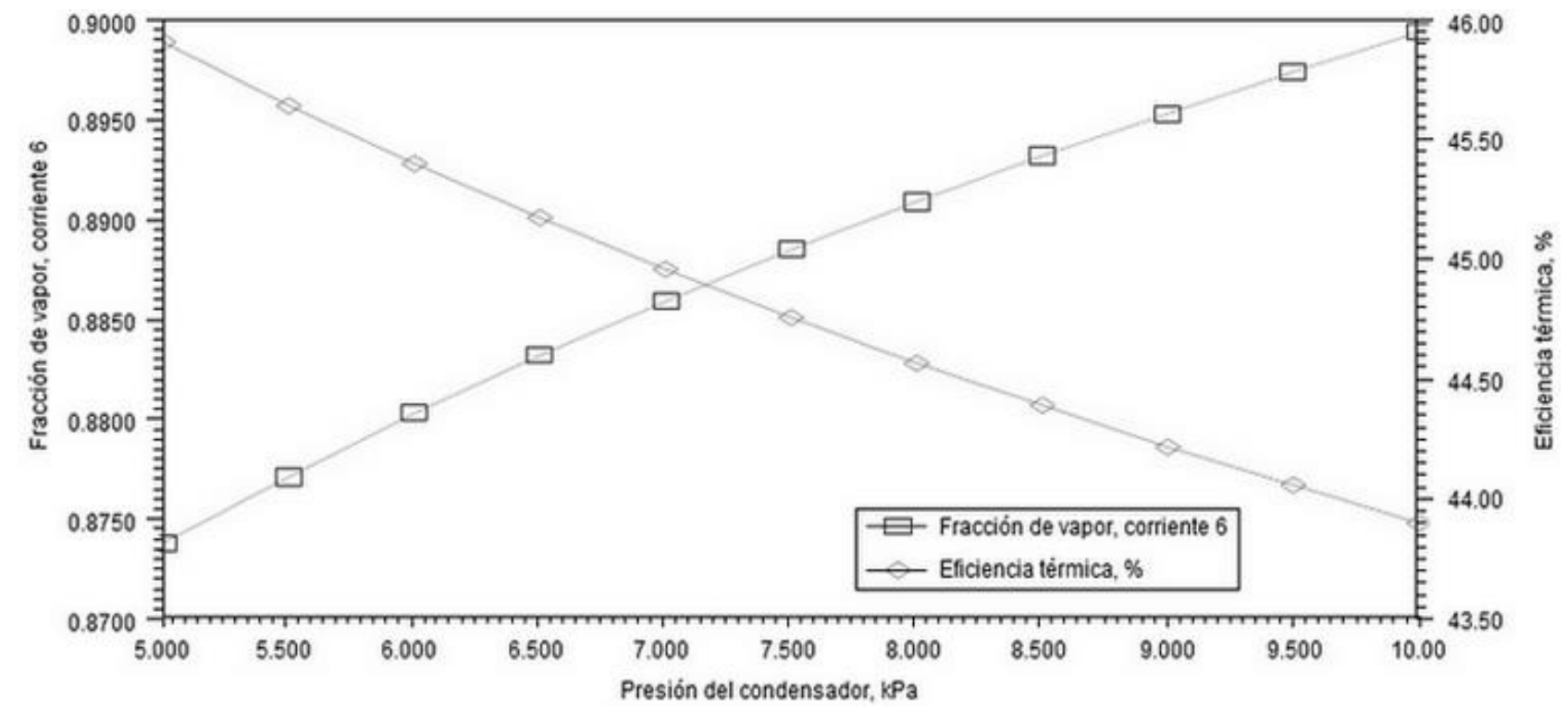

Fig. 8: Variación de la presión del condensador sobre la fracción de vapor y la eficiencia térmica del ciclo

\section{CONCLUSIONES}

El simulador Aspen HYSYS se adapta e interpreta correctamente el comportamiento del ciclo Rankine regenerativo con recalentamiento intermedio. También el simulador mostró confianza y eficiencia ante los cambios de las condiciones de operación, tales como; la variación de presión en el condensador y la fracción de vapor de salida de la turbina de baja presión sobre la eficiencia térmica del ciclo. Adicionalmente mostró resultados satisfactorios ante la influencia de la temperatura de entrada a la turbina sobre la eficiencia térmica del ciclo. Por lo tanto, los resultados muestran que el simulador Aspen HYSYS, a pesar de no ser un software direccionado para fines académicos, este muestra capacidad para ser usado y validar el ciclo Rankine regenerativo con recalentamiento intermedio. Además, el simulador se muestra como potencial herramienta para la enseñanza e interpretación de los ciclos de potencia de vapor en la disciplina de termodinámica. 


\section{REFERENCIAS}

Arce, P. y Vieira, N., Thermodynamics Simulation of Steam Power Cycles using GUI-MATLAB Interfaces, https//doi.org/10.9790/9622-0701038893, Int. Journal of Engineering Research and Application, 7(1), 88-93 (2017)

Arrieta, L., Valencia, G. Acevedo, C., Exergetic Evaluation of a Rankine Cycle with Regeneration: Effect of Turbine inlet Temperature and source Temperature, Int. Journal of Applied Engineering Research, 13(12), 10376-10380 (2018)

AspenTech, AspenONE® V7.3 Engineering Software, Bedford, Massachusetts 01730, USA (2011)

Brammer, N. y Hessami, M., Evaluation of a Process Simulator for Modelling and Analysis of Rankine Cycle Steam Power Plants, https://doi.org/10.1504/IJEX.2008.016674, International Journal of Exergy, 5(2), 177-192 (2008)

Cengel, Y. y Boles, M., Termodinámica, 7ª edición, 576-603. Mc Graw-Hill, México (2012)

Duarte, N., Valencia, G. y Arango, D., Theoretical Modeling of Power Cycles Using an Interactive Computational Tool, https://doi.org/10.21817/ijet/2018/v10i2/181002068, Int. Journal of Engineering and Technology, 10(2), 596-604 (2018)

Guerra, S., Vázquez, R. y Rodríguez, M., Simulación de Plantas de Cogeneración de Ciclo Combinado usando Aspen®, http://dx.doi.org/10.4067/S0718-07642005000100007, Información Tecnológica, 16(1), $42-49$ (2005)

Ibrahim, H., Elatrash, M. y Okasha, A., Steam Power Plant Design upgrading (case study: Khoms Steam Power Plant), http://dx.doi.org/10.5539/eer.v1n1p202, Energy and environment Research, 1(1), 202-211 (2011)

Liu, Z. y Karimi, I., Simulation of a Combined Cycle Gas Turbine Power Plant in Aspen HYSYS, https://doi.org/10.1016/j.egypro.2019.01.901, Energy Procedia, 158, 3620-3625 (2019)

Marwan, A., LImi, A. y Nurul, S., MATLAB as a Tool for the Teaching of Rankine Cycle with Simulation of a Simple Steam Power Plant, https://doi.org/10.11113/jt.v77.6781, Jurnal Teknologi, 77(28), 23-26 (2015)

Méndez, L., Salazar, M., Bonilla, A. y Lugo, R., Análisis Termodinámico de las Turbinas de Vapor para los Ciclos Ultracríticos, Supercríticos, Subcríticos y Geotérmicos, http://dx.doi.org/10.4067/S0718-07642019000400237, Información Tecnológica, 30(4), 237-248 (2019)

Mogollón, C., Ruiz, J., Bracho, M. y Villamar, C., Programa de Simulación de Ciclos Termodinámicos de Plantas de Vapor, Ciencia e Ingeniería, 28(3), 175-181 (2007)

Prosun, R., Analysis of Rankine Cycle and its Utility in Thermal Power Plant - a Theoretical Approach, International Journal of Mechanical and Production Engineering, 3(11), 53-59 (2015)

Rashidi, M., Aghagoli, A. y Ali, M., Thermodynamic Analysis of a Steam Power Plant with Double Reheat and Feed Water Heaters, https://doi.org/10.1155/2014/940818, Advances in Mechanical Engineering, 6, 1-11 (2014)

Rolle, K., Termodinámica, $6^{\text {ta }}$ edición, 405-411. Pearson-Prentice Hall, México (2006)

Sahu, C., Sao, M. y Jain, K., Comparison between Regenerative, Reheat \& Cogeneration Steam Plant on the basis of Turbine inlet Temperature, International Journal of Advanced Research in Science, Engineering and Technology, 4(1), 3270-3279 (2017)

Valderrama, J. y Véliz, C., Simulación de Ciclos de Vapor con Cogeneración usando una Ecuación de Estado, Información Tecnológica, 14(4), 29-38 (2003)

Valencia, G., Garcia, Y. y Diaz, D., Energy and Exergetic Analysis of a Regenerative Rankine Cycle with Feed Water Heaters, International Journal of ChemTech Research, 10(12), 36-44 (2017)

Valencia, G., Obregón, L. y Duarte, J., First-Law-Based Thermodynamic Study of Reheat and Regeneration Rankine Cycles Using an Educational Software, https://doi.org/10.12988/ces.2018.83104, Contemporary Engineering Sciences, 11(26), 1253-1260 (2018)

Wiser, W., Energy Resources: occurrence, production, conversion, use, technology, $1^{\mathrm{a}}$ edición, 189-190. Springer, New York (2000) 